\title{
MEADOW COMMUNITIES WITH CIRSIUM CANUM (L.) ALL. IN THE WIELOPOLKA RIVER VALLEY IN THE STRZYŻÓW FOOTHILLS (WESTERN CARPATHIANS)
}

\author{
Tomasz Wójcik, MaŁgorzata KotaŃSKA
}

T. Wójcik, Department of Plant Ecology, Jagiellonian University, Lubicz 46, 31-512 Kraków, Poland, e-mail: antomi7@wp.pl

M. Kotańska, Department of Environmental Biology, University of Rzeszów, Zelwerowicza 4, 35-601 Rzeszów, e-mail: mkotanska@o2.pl

(Received: January 9, 2014. Accepted: February 20, 2014)

\begin{abstract}
The research was conducted in meadow communities with Cirsium canum in the Wielopolka River valley in the Strzyżów Foothills in 2012-2013. The common phytosociological Braun-Blanquet method was applied and 20 phytosociological relevés were taken in each of the managed and abandoned patches. The aim of the study was to investigate the distribution of the species and identify the floristic composition and habitat conditions in the phytocoenoses, as well as to compare mown and unmown patches in which the species occurs. It was observed that the management factor exerted a significant effect on the floristic composition of the communities with Cirsium canum. The managed patches exhibited a large proportion of species from fresh and wet meadows (Molinio-Arrhenatheretea class), whereas the abandoned patches were clearly dominated by species from wet meadows from the Calthion alliance and Molinietalia order.
\end{abstract}

KEY WORDS: Cirsium canum, Calthion, Molinietalia, management, species diversity, Ellenberg's indicators, Strzyżów Fooothills

\section{INTRODUCTION}

In the Polish flora, Cirsium canum represents the Pontic-Pannonian element reaching the northern boundary of its occurrence range (ZAJĄC \& Z ZAJĄC 2009). The compact distribution range comprises central and eastern Europe. The species grows in northern Italy, Austria, the Czech Republic, Saxony, Poland, and Russia and reaches the Ural Mountains. Isolated localities of the species have been reported from Albania, the Caucasus, and Turkey (Meusel \& JÄGER 1992). In Poland, the species occurs mainly in the south of the country in the valleys of rivers and waterways. It has been frequently reported from the macroregions of the Silesia Lowland, Nida Basin, Kielce Upland, Western Polesie, Volhynian Polesie, Volhynian Upland, Sandomierz Basin, and Środkowobeskidzkie Foothills. Single and dispersed localities of the species have also been found in the western part of the Polish Lowland (ZAJĄC \& ZAJĄC 2001).
Cirsium canum is a perennial plant reaching a height of $1.5 \mathrm{~m}$. It is distinguished from other Cirsium species by oblong-lanceolate, grey-green, arachnoid hairy, dentate, and unevenly spinulose-bristled leaves. Purple-coloured flowers develop from June to September. The fruit is a pappus-bearing achene (RUTKOWsKi 2004).

The species is a hemicryptophyte with high light and thermal requirements. It prefers wet, eutrophic, neutral, or alkaline soils. It grows on wet meadows, pastures, and in ditches and along water banks (ZARZYCKI et al. 2002, RUTKOWSKI 2004).

Cirsium canum is classified by researchers into different taxonomic units within the Molinio-Arrhenatheretea class. As reported by Matuszkiewicz (2005), it represents species characteristic for the Calthion alliance of the Molinietalia order.

Preliminary field studies revealed quantitative and qualitative differences in the occurrence of the species within different meadow patches. 
The aim of the study was to investigate the distribution of $C$. canum in the Wielopolka River valley in the Strzyżów Foothills (a rare species in this region) and identify the floristic composition and habitat conditions of phytocoenoses in which the species occurs. Another objective was to compare the floristic composition of mown and unmown stands.

\section{STUDY AREA}

According to the physical-geographical division of Poland (Kondracki 2011), the study area belongs to the Strzyżów Foothills mesoregion, Środkowobeskidzkie Foothills macroregion, and the Outer Western Carpathians Subprovince. In the geobotanical division, it belongs to the Western Carpathians Division and Foothills Region (Pawıowski 1977 b). A more detailed division proposed by TowPAsz (1990) regards the study area as a separate Strzyżów-Dynów Foothills sub-region. TowPASz $(1987,1990)$ carried out research in the region and provided a report on the flora and geobotanical characteristics of the Strzyżów Foothills.

The study area covers the Wielopolka River valley, which stretches over a distance of ca. $20 \mathrm{~km}$ from Nawsie in the south to Ropczyce in the north. During heavy rainfall, the Wielopolka River valley is exposed to waterlogging; therefore, it mostly comprises grasslands with extensive forms of management, e.g. mowing or animal grazing, although wastelands and spontaneous riparian scrubs can also be found. The valley comprises the largest meadow and pasture complexes in the area of the Strzyżow Foothills, which have not been subjected to comprehensive phytosociological analyses so far. The meadow communities are dominated by fresh, wet, and sedge meadows; there are also rush communities.

\section{MATERIALS AND METHODS}

The investigations were conducted in the Wielopolka River valley in 2011-2013. In phytocoenoses comprising C. canum, 40 phytosociological relevés i.e. 20 relevés in mown and 20 in unmown stands were taken using the Braun-Blanquet method (PAwŁOWsKr 1977 a), and then they were compared in tables and analysed in detail. Phytosociological stability and the coverage coefficient were calculated for each relevé. The species names were given according to a checklist of vascular plants of Poland (Mirek et al. 2002). Phytosociological classification of the communities was taken from the guide of Matuszkiewicz (2005). The Shannon-Wiener index of diversity $\left(\mathrm{H}^{\prime}\right)$ was calculated as well (KREBS 2011). Using the phytoindication method developed by ElLENBERG et al. (1992), the values of indicators of insolation, thermal conditions, soil moisture, reaction, and trophy were calculated.

\section{RESULTS}

Cirsium canum is a rare species in the Wielopolka River valley. It occurs in four localities: Łączki Kucharskie, Niedźwiada, Borek Wielki, and Okonin. It grows in both managed and abandoned meadow phytocoenoses. In the mowed stands, it rarely exceeds the abundance values of 2-3 degrees and occurs single or in small clusters, whereas in abandoned stands it is often a dominant species growing in larger groups and achieving abundance values of $3-4$. The mown stands (Table 1) comprised between 19 and 39 species per relevé, while 11-26 species were recorded in the unmown patches (Table 2). In total, 64 species, out of which 26 exhibited stability degree IV-V, were noted in the mown patches. The other group of phytocoenoses comprised 71 species, but a majority occurred sporadically and only five species exhibited a high degree of stability (IV-V). The Shannon-Wiener index of diversity calculated for both groups of phytocoenoses revealed considerable differences. It reached a value of 3.003 in the mown stands and 2.717 in the abandoned stands.

In the mown phytocoenoses, a high stability degree $(\mathrm{V})$ is exhibited by Cirsium rivulare from the Calthion alliance, Equisetum palustre from the Molinietalia order, and Ranunculus repens and Lysimachia nummularia from the Agropyro-Rumicion crispi alliance. Stability degree $\mathrm{V}$ is also exhibited by species characteristic for the Molinio-Arrhenatheretea class: Holcus lanatus, Ranunculus acris, Trifolium pratense, Plantago lanceolata, and Festuca pratensis. Species from the Arrhenatherion association: Galium mollugo, and Geranium pratense and from the Arrhenatheretalia order: Trifolium repens, Leucanthemum vulgare, Dactylis glomerata, and Heracleum sphondylium are frequent and abundant species (stability degree IV). Other frequently occurring species included Symphytum officinale and Anthoxanthum odoratum, although they were not very abundant.

In the abandoned phytocoenoses, such a high degree of stability is achieved by Deschampsia caespitosa (V) and Equisetum palustre (IV) from the Molinietalia order, Carex hirta (V) from the Agropyro-Rumicion crispi alliance, and Carex gracilis (IV) from the Phragmitetea class. This community shows a high proportion of Deschampsia caespitosa, which is a co-dominant species together with C. canum. A higher abundance value is shown by rush species (Carex gracilis, Phalaris arundinacea). There are also species of the Artemisietea class, including taxa in the rank of invasive kenophytes (Solidago gigantea, Impatiens glandulifera). Particularly noteworthy is the occurrence of rare species for the region in this community, i.e. Dactylorhiza majalis, Polygonum bistorta, and Sanguisorbia officinalis.

Analysis of the share of species characteristic for the particular syntaxa revealed that the managed phytocoenoses exhibited a higher proportion of 
Table 1. Occurrence of Cirsium canum in managed meadow communities

\begin{tabular}{|c|c|c|c|c|c|c|c|c|c|c|c|c|c|c|c|c|c|c|c|c|c|c|}
\hline Succesive number of relevé & 1 & 2 & 3 & 4 & 5 & 6 & 7 & 8 & 9 & 10 & 11 & 12 & 13 & 14 & 15 & 16 & 17 & 18 & 19 & 20 & \multirow{8}{*}{ 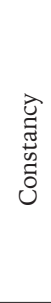 } & \multirow{8}{*}{ 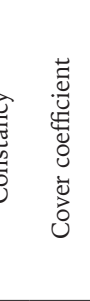 } \\
\hline Number of relevé in the field & 13 & 84 & 86 & 89 & 127 & 128 & 129 & 130 & 131 & 132 & 133 & 134 & 135 & 136 & 137 & 138 & 139 & 141 & 142 & 143 & & \\
\hline (day) & 20 & 9 & 9 & 9 & 21 & 21 & 21 & 21 & 21 & 21 & 21 & 21 & 21 & 21 & 22 & 22 & 22 & 22 & 22 & 22 & & \\
\hline \multirow[t]{2}{*}{ Date } & 5 & 8 & 8 & 8 & 5 & 5 & 5 & 5 & 5 & 5 & 5 & 5 & 5 & 5 & 5 & 5 & 5 & 5 & 5 & 5 & & \\
\hline & 2012 & 2012 & 2012 & 2012 & 2013 & 2013 & 2013 & 2013 & 2013 & 32013 & 2013 & 2013 & 2013 & 2013 & 2013 & 2013 & 2013 & 2013 & 2013 & 2013 & & \\
\hline Herb layer cover (\%) & 100 & 100 & 100 & 100 & 100 & 100 & 100 & 100 & 100 & 100 & 100 & 100 & 100 & 100 & 100 & 100 & 100 & 100 & 100 & 100 & & \\
\hline Management & $\mathrm{mII}$ & $\mathrm{mI}$ & $\mathrm{mI}$ & $\mathrm{mI}$ & $\mathrm{mII}$ & $\mathrm{mII}$ & $\mathrm{mII}$ & $\mathrm{mII}$ & $\mathrm{mI}$ & $\mathrm{mII}$ & $\mathrm{mII}$ & $\mathrm{mII}$ & $\mathrm{mII}$ & $\mathrm{mII}$ & $\mathrm{mII}$ & $\mathrm{mII}$ & $\mathrm{mII}$ & $\mathrm{mII}$ & $\mathrm{mII}$ & $\mathrm{mII}$ & & \\
\hline Number of species & 26 & 19 & 19 & 22 & 28 & 38 & 29 & 35 & 31 & 36 & 28 & 30 & 29 & 36 & 31 & 35 & 39 & 34 & 28 & 35 & & \\
\hline Cirsium canum & 1.2 & +.2 & +.2 & 2.2 & 1.3 & 1.2 & 3.4 & 1.2 & 3.3 & 3.3 & 4.5 & 1.2 & 2.3 & 1.2 & 2.3 & 3.4 & +.2 & 2.3 & 2.3 & + & $\mathrm{V}$ & 1,66 \\
\hline \multicolumn{23}{|l|}{ ChAll. Calthion } \\
\hline Cirsium rivulare & 3.3 & 2.2 & 2.2 & + & 1.3 & 1.2 & 1.2 & 2.2 & 1.2 & + & . & 1.2 & 2.3 & + & 1.2 & 2.3 & 1.2 & 1.2 & 3.3 & 3.3 & $\mathrm{~V}$ & 1,207 \\
\hline Trifolium hybridum & + & 2.2 & . & + & . & 1.2 & . & . & $\cdot$ & . & $\cdot$ & $\cdot$ & $\cdot$ & . & $\cdot$ & $\cdot$ & $\cdot$ & $\cdot$ & $\cdot$ & $\cdot$ & I & 117 \\
\hline Cirsium oleraceum & . & $\cdot$ & + & . & . & $\cdot$ & . & +.2 & . & . & 3.4 & . & . & . & . & . & . & . & . & . & I & 192 \\
\hline \multicolumn{23}{|l|}{ ChO. Molinietalia } \\
\hline Equisetum palustre & 1.1 & + & . & . & + & 1.1 & 1.1 & 1.1 & 1.1 & + & + & 2.1 & + & + & + & + & . & + & + & + & $\mathrm{V}$ & 240 \\
\hline Angelica sylvestris & + & . & . & . & . & $\cdot$ & $\cdot$ & + & + & + & + & + & + & . & . & $\mathrm{r}$ & . & $\mathrm{r}$ & . & + & III & 2 \\
\hline Lychnis flos-cuculi & + & + & . & . & . & . & . & + & + & + & . & . & . & + & r & $\mathrm{r}$ & . & . & r & + & III & 2 \\
\hline Deschampsia caespitosa & . & . & . & 1.2 & . & + & + & . & . & . & + & . & + & . & . & + & + & . & $\cdot$ & + & II & 4 \\
\hline Filipendula ulmaria & + & . & . & $\cdot$ & . & . & . & . & . & . & . & . & . & . & . & . & . & + & 1.3 & . & I & \\
\hline Lythrum salicaria & . & . & + & . & . & . & . & . & . & . & . & . & . & . & . & . & . & . & . & . & I & \\
\hline
\end{tabular}

ChAll. Arrhenatherion

\begin{tabular}{|c|c|c|c|c|c|c|c|c|c|c|c|c|c|c|c|c|c|c|c|c|c|c|}
\hline Galium mollugo & + & . & . & + & + & + & . & . & + & + & 1.2 & + & + & + & + & + & + & + & + & + & IV & 62 \\
\hline Geranium pratense & + & + & 1.2 & + & 1.2 & + & . & . & . & . & + & . & + & + & 1.2 & 1.2 & 1.2 & 3.3 & + & + & IV & 335 \\
\hline Crepis biennis & . & . & . & + & + & . & . & . & . & + & . & . & . & + & . & + & + & . & . & . & II & 15 \\
\hline Arrhenatherum elatius & . & 1.2 & 1.2 & 2.2 & . & . & . & . & . & . & . & . & + & . & . & . & +.2 & . & . & . & II & 142 \\
\hline
\end{tabular}

ChO. Arrhenatheretalia elatioris

Trifolium repens

Leucanthemum vulgare

Dactylis glomerata

Heracleum sphondylium

Taraxacum officinale

Lotus corniculatus

Pimpinella major

Achillea millefolium

Bellis perennis

Bromus hordeaceus

Carum carvi

Trifolium dubium

ChO. Trifolio fragiferae-Agrostietalia stoloniferae

Ranunculus repens

Lysimachia nummularia

Carex hirta

Mentha longifolia

Potentilla reptans

$\mathrm{ChCl}$. Molinio-Arrhenatheretea

\begin{tabular}{|c|c|c|c|c|c|c|c|c|c|c|c|c|c|c|c|c|c|c|c|c|c|c|}
\hline Holcus lanatus & 3.2 & + & + & . & 3.3 & 3.3 & 3.3 & 3.3 & 2.2 & 2.2 & 1.2 & 2.2 & 2.2 & 3.2 & 3.3 & 3.3 & 2.2 & 1.2 & 1.2 & 2.2 & $\mathrm{~V}$ & 2,105 \\
\hline Ranunculus acris & 3.3 & + & . & + & 1.2 & 1.2 & . & 1.2 & 2.2 & 2.2 & 1.2 & 2.2 & + & 1.2 & 2.2 & 2.2 & 1.2 & 2.2 & 2.2 & 2.2 & $\mathrm{~V}$ & 1,045 \\
\hline Trifolium pratense & 1.2 & . & . & 3.2 & 1.2 & + & 1.2 & 1.2 & + & 1.2 & + & 2.2 & + & 1.2 & 2.2 & 1.2 & + & 2.2 & + & 1.2 & $\mathrm{~V}$ & 665 \\
\hline Plantago lanceolata & . & + & + & 2.2 & + & + & + & + & + & + & + & + & + & 1.2 & 1.2 & + & + & + & . & + & V & 175 \\
\hline Festuca pratensis & 1.2 & . & . & . & 2.2 & 2.2 & 3.3 & 3.3 & 3.3 & 3.3 & 1.2 & 2.2 & 3.2 & 2.2 & 2.2 & 2.2 & 3.2 & 2.2 & 1.2 & 2.2 & V & 1,900 \\
\hline Alopecurus pratensis & 2.2 & . & . & . & 2.2 & + & 1.2 & + & 1.2 & + & + & . & 2.2 & + & 2.2 & 2.2 & + & 1.2 & 2.2 & 1.2 & IV & 640 \\
\hline Lathyrus pratensis & 2.3 & 1.2 & 3.2 & + & 1.2 & 1.2 & . & + & + & + & + & + & 2.2 & . & + & + & . & + & 2.2 & . & IV & 547 \\
\hline Poa pratensis & . & . & . & . & + & + & + & 1.2 & 1.2 & 1.2 & + & 1.2 & 1.2 & 1.2 & + & + & + & + & 1.2 & + & IV & 197 \\
\hline Rumex acetosa & 1.2 & + & . & . & . & . & + & + & + & + & + & . & + & + & + & + & + & + & + & + & IV & 60 \\
\hline Cerastium holosteoides & . & . & . & . & + & + & + & + & + & + & . & + & + & + & + & + & + & + & $\mathrm{r}$ & + & IV & 37 \\
\hline Alchemilla pastoralis & . & . & . & . & + & + & + & + & + & + & . & . & . & + & + & + & + & + & + & $\mathrm{r}$ & IV & 32 \\
\hline Centaurea jacea & . & + & . & + & . & +.2 & . & + & + & . & . & + & . & . & . & . & 1.2 & 1.2 & . & . & II & 65 \\
\hline Festuca rubra & . & . & . & . & . & + & . & . & . & . & + & . & . & . & . & . & . & + & + & . & I & 10 \\
\hline
\end{tabular}


Table 1. cont.

\begin{tabular}{|c|c|c|c|c|c|c|c|c|c|c|c|c|c|c|c|c|c|c|c|c|c|c|}
\hline Succesive number of relevé & 1 & 2 & 3 & 4 & 5 & 6 & 7 & 8 & 9 & 10 & 11 & 12 & 13 & 14 & 15 & 16 & 17 & 18 & 19 & 20 & & \\
\hline Number of relevé in the field & 13 & 84 & 86 & 89 & 127 & 128 & 129 & 130 & 131 & 132 & 133 & 134 & 135 & 136 & 137 & 138 & 139 & 141 & 142 & 143 & & \\
\hline (day) & 20 & 9 & 9 & 9 & 21 & 21 & 21 & 21 & 21 & 21 & 21 & 21 & 21 & 21 & 22 & 22 & 22 & 22 & 22 & 22 & & 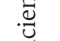 \\
\hline \multirow[t]{2}{*}{ (month) } & 5 & 8 & 8 & 8 & 5 & 5 & 5 & 5 & 5 & 5 & 5 & 5 & 5 & 5 & 5 & 5 & 5 & 5 & 5 & 5 & & 蛋 \\
\hline & 2012 & 2012 & 2012 & 2012 & 2013 & 2013 & 2013 & 2013 & 2013 & 2013 & 2013 & 2013 & 2013 & 2013 & 2013 & 2013 & 2013 & 2013 & 2013 & 2013 & $\tilde{0}$ & \\
\hline \multirow{2}{*}{$\begin{array}{l}\text { Herb layer cover (\%) } \\
\text { Management }\end{array}$} & 100 & 100 & 100 & 100 & 100 & 100 & 100 & 100 & 100 & 100 & 100 & 100 & 100 & 100 & 100 & 100 & 100 & 100 & 100 & 100 & & \\
\hline & $\mathrm{mII}$ & $\mathrm{mI}$ & $\mathrm{mI}$ & $\mathrm{mI}$ & $\mathrm{mII}$ & $\mathrm{mII}$ & $\mathrm{mII}$ & $\mathrm{mII}$ & $\mathrm{mI}$ & mII & $\mathrm{mII}$ & $\mathrm{mII}$ & mII & $\mathrm{mII}$ & $\mathrm{mII}$ & $\mathrm{mII}$ & $\mathrm{mII}$ & $\mathrm{mII}$ & mII & $\mathrm{mII}$ & & \\
\hline Number of species & 26 & 19 & 19 & 22 & 28 & 38 & 29 & 35 & 31 & 36 & 28 & 30 & 29 & 36 & 31 & 35 & 39 & 34 & 28 & 35 & & \\
\hline Phleum pratense & . & + & + & + & . & . & . & . & . & . & . & . & . & . & . & . & . & . & . & . & I & 7 \\
\hline Plantago major & . & . & . & . & . & + & . & + & . & . & . & . & . & . & . & . & . & . & . & . & I & 5 \\
\hline Poa trivialis & . & . & . & . & . & . & + & . & . & . & + & . & . & . & . & . & . & . & . & . & I & 5 \\
\hline Juncus tenuis & . & . & . & . & . & . & + & . & . & . & . & . & . & . & . & . & . & . & . & . & I & 2 \\
\hline Leontodon hispidus & . & . & . & . & . & . & . & . & . & . & . & . & . & . & . & . & + & . & . & . & I & 2 \\
\hline \multicolumn{23}{|l|}{$\mathrm{ChCl}$. Artemisietea vulgaris } \\
\hline Glechoma hederacea & . & + & . & . & . & + & + & . & + & + & + & . & + & + & + & + & + & + & . & + & IV & 32 \\
\hline Galium aparine & + & . & . & . & . & . & . & . & . & . & . & . & + & . & . & . & . & . & + & . & I & 7 \\
\hline \multicolumn{23}{|l|}{ ChCl. Phragmitetea } \\
\hline Phalaris arundinacea & . & . & + & + & . & . & . & + & . & + & + & . & . & . & . & + & + & . & . & . & II & 17 \\
\hline Carex gracilis & + & 3.3 & 2.2 & . & . & . & . & . & . & . & . & . & . & . & . & . & . & . & . & + & I & 280 \\
\hline Carex vulpina & +.2 & $\cdot$ & . & . & . & . & . & . & . & . & . & . & . & . & . & & . & . & . & + & I & 5 \\
\hline \multicolumn{23}{|l|}{ Others } \\
\hline Symphytum officinale & + & +.2 & + & + & + & + & +.2 & +.2 & . & & 1.2 & +.2 & + & + & + & + & + & . & 1.2 & + & $\mathrm{V}$ & 87 \\
\hline Anthoxanthum odoratum & . & . & . & . & . & 1.1 & + & 1.2 & + & + & + & + & + & 1.1 & . & + & + & + & + & + & IV & 102 \\
\hline Polygonum amphibium & + & . & . & . & + & + & + & . & + & + & . & + & + & . & + & + & + & + & . & . & III & 30 \\
\hline Ajuga reptans & . & . & . & . & $\mathrm{r}$ & + & . & . & . & + & . & . & . & + & $\mathrm{r}$ & . & + & . & . & $\mathrm{r}$ & II & 17 \\
\hline Ranunculus auricomus & . & . & . & . & . & . & . & . & . & . & + & . & + & + & . & 1.2 & + & . & . & + & II & 37 \\
\hline Luzula campestris & . & . & . & . & + & + & . & + & . & . & . & . & . & + & . & . & $\mathrm{r}$ & . & . & . & II & 12 \\
\hline Lolium multiflorum & . & . & 1.2 & 2.2 & . & . & + & . & & . & . & . & . & . & . & . & . & . & . & & I & 115 \\
\hline Briza media & . & . & . & . & . & + & . & + & . & . & . & . & . & . & . & . & . & . & . & . & I & 5 \\
\hline Anemone nemorosa & . & . & . & . & . & +.2 & . & . & . & . & . & . & . & . & . & . & . & . & . & . & I & 2 \\
\hline Ficaria verna & . & . & & . & . & . & . & . & & . & . & . & . & + & . & . & . & . & . & & I & 2 \\
\hline Vicia sepium & . & . & . & . & . & + & . & . & . & . & . & . & . & . & . & . & . & . & . & . & I & 2 \\
\hline
\end{tabular}

Explanations:

Management: $\mathrm{mII}$ - twice movn meadow, $\mathrm{mI}$ - once movn meadow.

Table 2. Occurrence of Cirsium canum in abandoned meadow communities

\begin{tabular}{|c|c|c|c|c|c|c|c|c|c|c|c|c|c|c|c|c|c|c|c|c|c|c|}
\hline Succesive number of relevé & 1 & 2 & 3 & 4 & 5 & 6 & 7 & 8 & 9 & 10 & 11 & 12 & 13 & 14 & 15 & 16 & 17 & 18 & 19 & 20 & \multirow{8}{*}{ 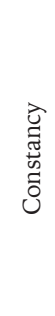 } & \multirow{8}{*}{ 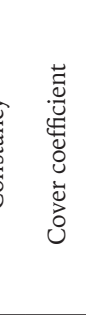 } \\
\hline Number of relevé in the field & 61 & 88 & 148 & 149 & 151 & 209 & 210 & 211 & 212 & 214 & 232 & 233 & 234 & 235 & 236 & 237 & 238 & 239 & 240 & 241 & & \\
\hline (day) & 6 & 9 & 22 & 22 & 22 & 29 & 29 & 29 & 29 & 29 & 19 & 19 & 19 & 19 & 19 & 19 & 19 & 19 & 19 & 19 & & \\
\hline \multirow[t]{2}{*}{ (month) } & 8 & 8 & 5 & 5 & 5 & 7 & 7 & 7 & 7 & 7 & 8 & 8 & 8 & 8 & 8 & 8 & 8 & 8 & 8 & 8 & & \\
\hline & 2012 & 2012 & 2013 & 2013 & 2013 & 2013 & 2013 & 2013 & 2013 & 2013 & 2013 & 2013 & 2013 & 2013 & 2013 & 2013 & 2013 & 2013 & 2013 & 2013 & & \\
\hline Herb layer cover $(\%)$ & 100 & 100 & 100 & 100 & 100 & 100 & 100 & 100 & 100 & 100 & 100 & 100 & 100 & 100 & 100 & 100 & 100 & 100 & 100 & 100 & & \\
\hline Management & - & - & 2011 & 2011 & 2011 & - & - & - & - & - & - & - & - & - & - & - & - & - & - & - & & \\
\hline Number of species & 11 & 13 & 25 & 23 & 23 & 14 & 13 & 21 & 20 & 16 & 24 & 26 & 26 & 16 & 19 & 22 & 20 & 16 & 21 & 20 & & \\
\hline Cirsium canum & 4.4 & 3.3 & +.3 & 1.2 & + & 2.3 & 2.2 & 3.3 & 3.2 & 1.2 & 3.3 & 2.3 & 2.3 & 5.5 & 4.4 & 2.3 & 4.4 & 1.2 & 1.2 & 2.2 & $\mathrm{~V}$ & 2,755 \\
\hline \multicolumn{23}{|l|}{ ChAll. Calthion } \\
\hline Cirsium rivulare & + & + & 2.2 & 2.3 & 1.2 & . & + & 1.2 & +.2 & +.2 & . & . & . & . & +.2 & . & . & . & . & . & III & 240 \\
\hline Polygonum bistorta & . & . & . & . & . & . & . & . & . & . & + & + & + & + & + & + & 1.2 & 1.2 & 1.2 & 1.2 & III & 115 \\
\hline Epilobium palustre & . & . & . & . & $\cdot$ & . & . & . & . & + & . & + & 1.1 & + & + & + & $\cdot$ & 2.2 & 1.2 & 1.2 & III & 175 \\
\hline Caltha palustris & . & . & 2.2 & 1.2 & 2.2 & . & . & . & . & . & . & . & . & . & . & . & . & . & . & . & I & 200 \\
\hline Scirpus sylvaticus & . & . & . & . & + & + & . & . & 1.2 & . & . & . & . & . & . & . & . & . & . & . & I & 30 \\
\hline Dactylorhiza majalis & . & . & + & r & + & . & . & . & . & . & . & . & . & . & . & . & . & . & . & . & I & 7 \\
\hline
\end{tabular}


Table 2. cont.

\begin{tabular}{cccccccccccccccccccccc}
\hline Succesive number of relevé & 1 & 2 & 3 & 4 & 5 & 6 & 7 & 8 & 9 & 10 & 11 & 12 & 13 & 14 & 15 & 16 & 17 & 18 & 19 & 20 \\
Number of relevé in the field & 61 & 88 & 148 & 149 & 151 & 209 & 210 & 211 & 212 & 214 & 232 & 233 & 234 & 235 & 236 & 237 & 238 & 239 & 240 & 241 \\
& (day) & 6 & 9 & 22 & 22 & 22 & 29 & 29 & 29 & 29 & 29 & 19 & 19 & 19 & 19 & 19 & 19 & 19 & 19 & 19 & 19 \\
Date & (month) & 8 & 8 & 5 & 5 & 5 & 7 & 7 & 7 & 7 & 7 & 8 & 8 & 8 & 8 & 8 & 8 & 8 & 8 & 8 & 8 \\
& (year) & 2012 & 2012 & 2013 & 2013 & 2013 & 2013 & 2013 & 2013 & 2013 & 2013 & 2013 & 2013 & 2013 & 2013 & 2013 & 2013 & 2013 & 2013 & 2013 & 2013
\end{tabular}

Herb layer cover (\%) $\quad \begin{array}{lllllllllllllllllllll}100 & 100 & 100 & 100 & 100 & 100 & 100 & 100 & 100 & 100 & 100 & 100 & 100 & 100 & 100 & 100 & 100 & 100 & 100 & 100\end{array}$

Management

Number of species $\begin{array}{lllllllllllllllllllll}11 & 13 & 25 & 23 & 23 & 14 & 13 & 21 & 20 & 16 & 24 & 26 & 26 & 16 & 19 & 22 & 20 & 16 & 21 & 20\end{array}$

Myosotis palustris

Juncus effusus

ChO. Molinietalia

Deschampsia caespitosa

Equisetum palustre

Lythrum salicaria

Filipendula ulmaria

Selinum carvifolia

Lychnis flos-cuculi

Sanguisorbia officinalis

Angelica sylvestris

Cirsium palustre

Lotus uliginosus

Lysimachia vulgaris

Valeriana officinalis

ChAll. Arrhenatherion

Geranium pratense

Galium mollugo

Arrhenatherum elatius

Crepis biennis

ChO. Arrhenatheretalia elatioris

Achillea millefolium

Dactylis glomerata

Heracleum sphondylium

Daucus carota

Bromus hordeaceus

Taraxacum officinale

ChO. Trifolio fragiferae-Agrostietalia stoloniferae

Carex hirta

Rumex crispus

Ranunculus repens

Lysimachia nummularia

Mentha longifolia

Elymus repens

Juncus inflexus

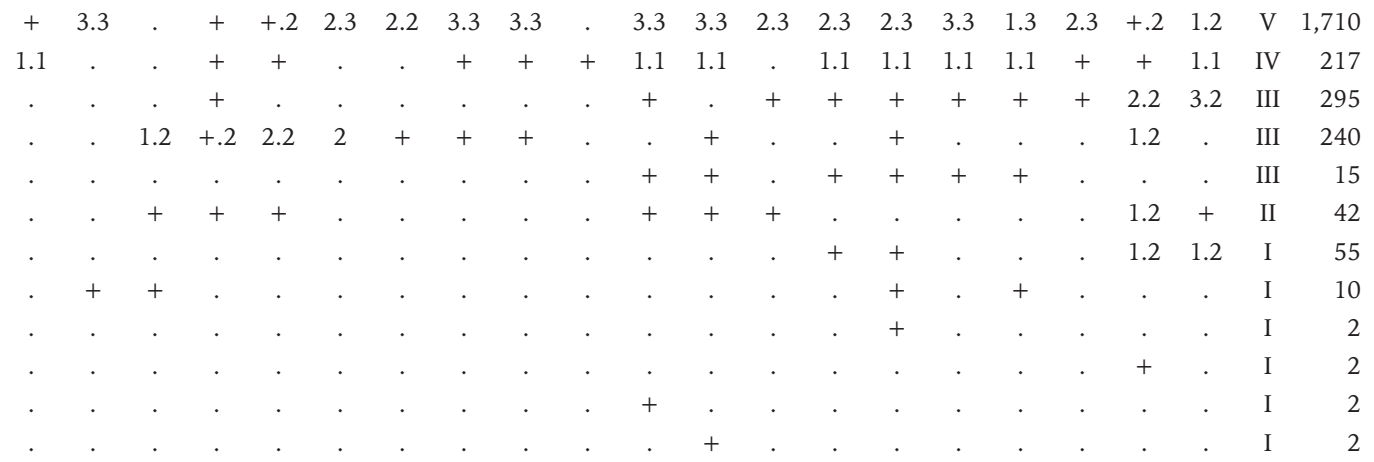

1.2

ChCl. Molinio-Arrhenatheretea

\begin{tabular}{|c|c|c|c|c|c|c|c|c|c|c|c|c|c|c|c|c|c|c|c|c|c|c|}
\hline Holcus lanatus & . & . & 1.2 & . & + & . & . & + & + & . & 1.2 & 2.2 & 2.2 & . & 1.2 & 1.2 & 1.2 & 2.2 & . & . & III & 332 \\
\hline Lathyrus pratensis & . & . & 1.2 & 1.2 & 1.2 & + & + & + & + & . & + & 2.2 & + & 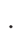 & . & + & . & . & . & . & III & 180 \\
\hline Plantago lanceolata & . & . & + & + & . & + & . & . & . & . & + & + & . & ${ }^{\circ}$ & . & . & . & + & + & + & II & 20 \\
\hline Alopecurus pratensis & . & . & 2.2 & 1.2 & 1.2 & + & + & 1.2 & 1.2 & . & . & . & . & 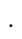 & . & . & . & . & . & . & II & 192 \\
\hline Festuca rubra & . & . & +.2 & . & + & . & . & . & . & . & + & 1.2 & 1.2 & $\cdot$ & . & +.2 & . & . & . & . & II & 60 \\
\hline Poa pratensis & . & + & 1.2 & 1.2 & . & . & . & 1.2 & + & . & . & . & . & & . & . & . & . & . & . & II & 80 \\
\hline Centaurea jacea & + & + & . & . & . & + & + & . & + & . & . & . & . & . & . & . & . & . & . & . & II & 12 \\
\hline Rumex acetosa & . & . & + & + & + & . & . & . & . & . & . & . & . & . & . & . & . & . & . & . & I & 7 \\
\hline Alchemilla pastoralis & . & . & . & + & + & . & . & . & . & . & . & . & . & • & . & . & . & . & . & . & I & 5 \\
\hline Cerastium holosteoides & . & . & . & . & . & . & . & . & . & . & . & + & + & . & . & . & . & . & . & . & I & 5 \\
\hline Prunella vulgaris & . & . & . & . & + & . & . & . & . & . & . & . & +.2 & - & . & . & . & . & . & . & I & 5 \\
\hline Ranunculus acris & . & . & + & + & . & . & . & . & . & . & . & . & . & . & . & . & . & . & . & . & I & 5 \\
\hline Festuca pratensis & . & . & 3.2 & . & . & . & . & . & . & . & . & . & . & . & . & . & . & . & . & . & $\mathrm{I}$ & 187 \\
\hline
\end{tabular}


Table 2. cont.

\begin{tabular}{|c|c|c|c|c|c|c|c|c|c|c|c|c|c|c|c|c|c|c|c|c|c|c|}
\hline Succesive number of relevé & 1 & 2 & 3 & 4 & 5 & 6 & 7 & 8 & 9 & 10 & 11 & 12 & 13 & 14 & 15 & 16 & 17 & 18 & 19 & 20 & \multirow{8}{*}{ 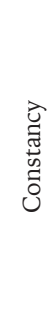 } & \multirow{8}{*}{ 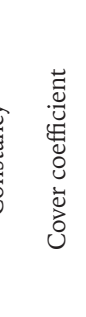 } \\
\hline Number of relevé in the field & 61 & 88 & 148 & 149 & 151 & 209 & 210 & 211 & 212 & 214 & 232 & 233 & 234 & 235 & 236 & 237 & 238 & 239 & 240 & 241 & & \\
\hline (day) & 6 & 9 & 22 & 22 & 22 & 29 & 29 & 29 & 29 & 29 & 19 & 19 & 19 & 19 & 19 & 19 & 19 & 19 & 19 & 19 & & \\
\hline \multirow[t]{2}{*}{ (month) } & 8 & 8 & 5 & 5 & 5 & 7 & 7 & 7 & 7 & 7 & 8 & 8 & 8 & 8 & 8 & 8 & 8 & 8 & 8 & 8 & & \\
\hline & 2012 & 2012 & 2013 & 2013 & 2013 & 2013 & 2013 & 2013 & 2013 & 2013 & 2013 & 2013 & 2013 & 2013 & 2013 & 2013 & 2013 & 2013 & 2013 & 2013 & & \\
\hline Herb layer cover $(\%)$ & 100 & 100 & 100 & 100 & 100 & 100 & 100 & 100 & 100 & 100 & 100 & 100 & 100 & 100 & 100 & 100 & 100 & 100 & 100 & 100 & & \\
\hline Management & - & - & 2011 & 2011 & 2011 & - & - & - & - & - & - & - & - & - & - & - & - & - & - & - & & \\
\hline Number of species & 11 & 13 & 25 & 23 & 23 & 14 & 13 & 21 & 20 & 16 & 24 & 26 & 26 & 16 & 19 & 22 & 20 & 16 & 21 & 20 & & \\
\hline Phleum pratense & . & . & . & . & . & . & . & . & . & . & + & + & . & . & . & + & . & . & . & . & I & 7 \\
\hline Poa trivialis & . & . & . & . & . & . & . & + & . & . & . & . & . & . & . & . & . & . & . & . & I & 2 \\
\hline Trifolium pratense & . & . & + & . & . & . & . & . & . & . & . & . & . & . & . & . & . & . & . & . & I & 2 \\
\hline \multicolumn{23}{|l|}{ ChCl. Artemisietea vulgaris } \\
\hline Cirsium arvense & . & . & . & . & . & . & . & . & . & . & + & . & 1.1 & + & . & 1.2 & + & + & + & 1.2 & II & 87 \\
\hline Galium aparine & . & + & . & . & . & . & 1.2 & + & 1.2 & + & . & . & . & . & . & . & + & . & + & 1.2 & II & 87 \\
\hline Solidago gigantea & . & . & . & . & . & . & . & +.2 & . & . & . & + & + & + & . & + & + & . & . & + & II & 17 \\
\hline Glechoma hederacea & . & . & . & . & . & . & . & . & . & + & . & . & + & . & . & + & + & . & . & . & I & 10 \\
\hline Impatiens glandulifera & . & . & . & . & . & . & . & . & . & . & . & . & . & . & . & + & + & + & . & . & I & 7 \\
\hline Artemisia vulgaris & . & . & . & . & . & . & . & . & . & + & . & . & . & + & . & . & . & . & . & . & I & 5 \\
\hline Calystegia sepium & . & . & . & . & . & + & . & . & + & . & . & . & . & . & . & . & . & . & . & . & I & 5 \\
\hline \multicolumn{23}{|l|}{ ChCl. Phragmitetea } \\
\hline Carex gracilis & . & 2.2 & + & 3.2 & 3.3 & 4.2 & 3.2 & 2.2 & 1.2 & 2.2 & + & + & . & . & . & + & 1.2 & 3.2 & 2.2 & . & IV & 1,473 \\
\hline Phalaris arundinacea & 3.2 & 1.2 & . & 1.2 & . & 1.2 & 2.2 & 1.2 & 2.2 & 3.2 & . & . & . & . & . & . & . & . & . & . & II & 650 \\
\hline Carex vulpina & . & . & + & +.2 & . & . & . & . & . & . & . & . & . & . & . & . & . & . & . & . & I & 5 \\
\hline Phragmites australis & . & . & . & . & . & . & . & . & . & . & . & . & . & . & . & . & . & . & + & . & I & 2 \\
\hline \multicolumn{23}{|l|}{ Others } \\
\hline Symphytum officinale & + & . & . & . & . & + & . & + & + & 1.2 & + & + & + & + & + & . & + & . & . & + & III & 52 \\
\hline Geum rivale & . & . & . & . & . & . & . & . & . & . & . & . & . & . & 1.2 & + & + & + & . & . & I & 32 \\
\hline Mentha arvensis & . & . & . & . & . & . & . & . & . & . & + & . & + & . & . & . & . & . & . & . & I & 5 \\
\hline Alnus glutinosa & . & . & . & . & . & . & . & . & . & . & . & . & . & . & . & . & . & . & + & + & I & 5 \\
\hline Urtica dioica & . & . & . & . & . & . & . & . & . & + & . & . & . & . & . & + & . & . & . & . & I & 5 \\
\hline Ajuga reptans & . & . & + & . & . & . & . & . & . & . & . & . & . & . & . & . & . & . & . & . & I & 2 \\
\hline Veronica chamaedrys & . & . & . & . & . & . & . & . & . & . & . & . & + & . & . & . & . & . & . & . & I & 2 \\
\hline
\end{tabular}

Explanations:

Management: 2011 - unmoving from 2011 year.

Table 3. Comparison of the floristic composition of (managed and abandoned) meadow communities with Cirsium canum in the Wielopolka River valley

\begin{tabular}{|c|c|c|c|c|}
\hline Management & \multicolumn{2}{|c|}{1} & \multicolumn{2}{|c|}{2} \\
\hline Number of relevés & \multicolumn{2}{|c|}{20} & \multicolumn{2}{|c|}{20} \\
\hline Total numer of species & \multicolumn{2}{|c|}{65} & \multicolumn{2}{|c|}{72} \\
\hline Mean number of species in 1 relevé & \multicolumn{2}{|c|}{30} & \multicolumn{2}{|c|}{19} \\
\hline Shannon-Wiener index $\left(\mathrm{H}^{\prime}\right)$ & \multicolumn{2}{|c|}{3.003} & \multicolumn{2}{|c|}{2.717} \\
\hline Cirsium canum & V & 1,660 & V & 2,755 \\
\hline \multicolumn{5}{|l|}{ Ch. Molinietalia (incl. Calthion*) } \\
\hline Cirsium rivulare* & V & 1,207 & III & 240 \\
\hline Equisetum palustre & $\mathrm{V}$ & 240 & IV & 217 \\
\hline Angelica sylvestris & III & 25 & I & 10 \\
\hline Lychnis flos-cuculi & III & 25 & II & 42 \\
\hline Deschampsia caespitosa & II & 42 & $\mathrm{~V}$ & 1,710 \\
\hline Filipendula ulmaria & I & 30 & III & 240 \\
\hline Lythrum salicaria & I & 2 & III & 295 \\
\hline
\end{tabular}


Table 3. cont.

\begin{tabular}{|c|c|c|c|c|}
\hline Management & \multicolumn{2}{|c|}{1} & \multicolumn{2}{|c|}{2} \\
\hline Number of relevés & \multicolumn{2}{|c|}{20} & \multicolumn{2}{|c|}{20} \\
\hline Total numer of species & \multicolumn{2}{|c|}{65} & \multicolumn{2}{|c|}{72} \\
\hline Mean number of species in 1 relevé & \multicolumn{2}{|c|}{30} & \multicolumn{2}{|c|}{19} \\
\hline Shannon-Wiener index $\left(\mathrm{H}^{\prime}\right)$ & \multicolumn{2}{|c|}{3.003} & \multicolumn{2}{|c|}{2.717} \\
\hline Polygonum bistorta* & & & III & 115 \\
\hline Epilobium palustre* & & & III & 175 \\
\hline Selinum carvifolia & & & III & 15 \\
\hline Caltha palustris* & & & I & 200 \\
\hline Sanguisorbia officinalis & & & I & 55 \\
\hline Scirpus sylvaticus* & & & I & 30 \\
\hline Dactylorhiza majalis* & & & I & 7 \\
\hline Myosotis palustris* & & & I & 5 \\
\hline Cirsium palustre & & & I & 2 \\
\hline Lotus uliginosus & & & I & 2 \\
\hline Lysimachia vulgaris & & & I & 2 \\
\hline Valeriana officinalis & & & I & 2 \\
\hline Juncus effusus* & & & I & 2 \\
\hline Trifolium hybridum* & I & 117 & & \\
\hline Cirsium oleraceum* & I & 192 & & \\
\hline \multicolumn{5}{|c|}{ Ch. Arrhenatheretalia (incl. Arrhenatherion*) } \\
\hline Trifolium repens & IV & 492 & & \\
\hline Leucanthemum vulgare & IV & 252 & & \\
\hline Lotus corniculatus & III & 50 & & \\
\hline Pimpinella major & III & 47 & & \\
\hline Bellis perennis & III & 25 & & \\
\hline Geranium pratense* & IV & 335 & III & 155 \\
\hline Dactylis glomerata & IV & 162 & II & 62 \\
\hline Galium mollugo* & IV & 62 & III & 47 \\
\hline Heracleum sphondylium & IV & 32 & II & 12 \\
\hline Taraxacum officinale & III & 225 & $\mathrm{I}$ & 2 \\
\hline Achillea millefolium & III & 25 & II & 65 \\
\hline Arrhenatherum elatius* & II & 142 & $\mathrm{I}$ & 5 \\
\hline Bromus hordeaceus & II & 15 & I & 2 \\
\hline Crepis biennis* & II & 15 & I & 5 \\
\hline Carum carvi & $\mathrm{I}$ & 10 & & \\
\hline Trifolium dubium & I & 2 & & \\
\hline Daucus carota & & & I & 5 \\
\hline \multicolumn{5}{|c|}{ Ch. Trifolio fragiferae-Agrostietalia stoloniferae } \\
\hline Ranunculus repens & $\mathrm{V}$ & 375 & II & 20 \\
\hline Lysimachia nummularia & $\mathrm{V}$ & 47 & II & 40 \\
\hline Carex hirta & IV & 32 & $\mathrm{~V}$ & 647 \\
\hline Rumex crispus & & & III & 22 \\
\hline Elymus repens & & & I & 27 \\
\hline Mentha longifolia & I & 2 & I & 7 \\
\hline Potentilla reptans & I & 2 & & \\
\hline Juncus inflexus & & & I & 2 \\
\hline \multicolumn{5}{|l|}{ Ch. Molinio-Arrhenatheretea } \\
\hline Holcus lanatus & $\mathrm{V}$ & 2,105 & III & 332 \\
\hline Ranunculus acris & $\mathrm{V}$ & 1,045 & I & 5 \\
\hline Trifolium pratense & $\mathrm{V}$ & 665 & I & 2 \\
\hline Plantago lanceolata & $\mathrm{V}$ & 175 & II & 20 \\
\hline
\end{tabular}


Table 3. cont.

\begin{tabular}{|c|c|c|c|c|}
\hline Management & \multicolumn{2}{|c|}{1} & \multicolumn{2}{|c|}{2} \\
\hline Number of relevés & \multicolumn{2}{|c|}{20} & \multicolumn{2}{|c|}{20} \\
\hline Total numer of species & \multicolumn{2}{|c|}{65} & \multicolumn{2}{|c|}{72} \\
\hline Mean number of species in 1 relevé & \multicolumn{2}{|c|}{30} & \multicolumn{2}{|c|}{19} \\
\hline Shannon-Wiener index $\left(\mathrm{H}^{\prime}\right)$ & \multicolumn{2}{|c|}{3.003} & \multicolumn{2}{|c|}{2.717} \\
\hline Festuca pratensis & $\mathrm{V}$ & 1,900 & I & 187 \\
\hline Alopecurus pratensis & IV & 640 & II & 192 \\
\hline Lathyrus pratensis & IV & 547 & III & 180 \\
\hline Poa pratensis & IV & 197 & II & 80 \\
\hline Rumex acetosa & IV & 60 & I & 7 \\
\hline Cerastium holosteoides & IV & 37 & I & 5 \\
\hline Alchemilla pastoralis & IV & 32 & I & 5 \\
\hline Centaurea jacea & II & 65 & II & 12 \\
\hline Festuca rubra & I & 10 & II & 60 \\
\hline Phleum pratense & I & 7 & I & 7 \\
\hline Poa trivialis & I & 5 & I & 2 \\
\hline Plantago major & I & 5 & & \\
\hline Juncus tenuis & I & 2 & & \\
\hline Leontodon hispidus & I & 2 & & \\
\hline Prunella vulgaris & & & I & 5 \\
\hline \multicolumn{5}{|l|}{ Ch. Artemisietea vulgaris } \\
\hline Glechoma hederacea & IV & 32 & I & 10 \\
\hline Galium aparine & I & 7 & II & 87 \\
\hline Cirsium arvense & & & II & 87 \\
\hline Solidago gigantea & & & II & 17 \\
\hline Impatiens glandulifera & & & I & 7 \\
\hline Artemisia vulgaris & & & I & 5 \\
\hline Calystegia sepium & & & I & 5 \\
\hline \multicolumn{5}{|l|}{ Ch. Phragmitetea } \\
\hline Carex gracilis & $\mathrm{I}$ & 280 & IV & 1,472 \\
\hline Phalaris arundinacea & II & 17 & II & 650 \\
\hline Carex vulpina & I & 5 & I & 5 \\
\hline Phragmites australis & & & I & 2 \\
\hline \multicolumn{5}{|l|}{ Others } \\
\hline Anthoxanthum odoratum & IV & 102 & & \\
\hline Polygonum amphibium & III & 30 & & \\
\hline Ranunculus auricomus & II & 37 & & \\
\hline Luzula campestris & II & 12 & & \\
\hline Lolium multiflorum & I & 115 & & \\
\hline Briza media & I & 5 & & \\
\hline Anemone nemorosa & I & 2 & & \\
\hline Ficaria verna & I & 2 & & \\
\hline Vicia sepium & I & 2 & & \\
\hline Symphytum officinale & $\mathrm{V}$ & 87 & III & 52 \\
\hline Ajuga reptans & II & 17 & I & 2 \\
\hline Geum rivale & & & I & 32 \\
\hline Mentha arvensis & & & I & 5 \\
\hline Alnus glutinosa & & & I & 5 \\
\hline Urtica dioica & & & I & 5 \\
\hline Veronica chamaedrys & & & I & 2 \\
\hline
\end{tabular}

Explanations:

Management: 1 - managed, 2 - abandoned. 
Table 4. Mean Ellenberg's indication values of L, T, F, R and $\mathrm{N}$ indices in the studied communities

\begin{tabular}{lccccc}
\hline \multicolumn{1}{c}{ Specification } & $\mathrm{L}$ & $\mathrm{T}$ & $\mathrm{F}$ & $\mathrm{R}$ & $\mathrm{N}$ \\
\hline Community with C. canum (managed) & 7.4 & 5.9 & 6.3 & 6.9 & 5.6 \\
Community with C. canum (abandoned) & 7.1 & 5.8 & 7.4 & 6.5 & 4.6 \\
\hline
\end{tabular}

Explanations: $\mathrm{L}$ - solar radiation, $\mathrm{T}$ - temperature radiation, $\mathrm{F}$ - soil moisture, $\mathrm{R}$ - soil reaction, $\mathrm{N}$ - soil nitrogen content.

species from the Arrhenatheretalia order and Molinio-Arrhenatheretea class, whereas the abandoned phytocoenoses had a higher share of species from the Calthion alliance Molinietalia order, and Artemisietea and Phragmitetea classes (Table 3).

The mean values of Ellenberg's environmental indicators show that both groups of phytocoenoses occupied well-insolated, moderately warm habitats with neutral or slightly acidic reaction of soils (Table 4). The mown phytocoenoses covered fresh and moderately moist soils, whereas the unmowed phytocoenoses grew on wet and highly wet soils. Analysis of the nitrogen level indicator revealed that the unmown stands were found at the border of poor or moderately poor habitats. In turn, the managed patches exhibited considerably higher substrate fertility between mesotrophic and eutrophic habitats.

\section{DISCUSSION}

According to the generally adopted classification of plant communities proposed by MatuszKIEWICZ (2005), C. canum is regarded to be characteristic for the Calthion alliance. FijaŁKowski \& CHojnacka-FijatKowsKA (1990) also classify the species into the group of wet meadows. The authors described the Cirsietum cani association in the Lublin region, which exhibited dominance of species characteristic for the Molinietalia order. KotAŃSKA \& TowPAsz (2007) analysed phytocoenoses with $C$. canum on the Proszowice Plateau, and classified this species into the Arrhenatherion alliance and Arrhenatheretalia elatioris order after calculating the systematic value of species groups (PAwŁowski 1977 a). Meadow communities with C. canum were similarly classified by VICHEREK (1962), who investigated the occurrence thereof in Moravia.

The results of the study conducted in the meadow communities with C. canum in the Wielopolka River valley indicate that the species is difficult to classify into a specific syntaxonomic unit. An additional difficulty is posed by the form of management of the investigated phytocoenoses. In the mowed patches, a high proportion is constituted by fresh meadow species, which are characterised by a greater number and species coverage than wet meadow species. A high share and stability degree are exhibited by species characteristic for the Molinio-Arrhenatheretea class. This is undoubtedly related to regular mowing and, probably, fertilisation and sowing, which have an effect on species diversity. Therefore, the mown phytocoenoses with the share of $C$. canum repre- sent an intermediate community between the Calthion and Arrhenatherion alliances.

The abandoned phytocoenoses are clearly dominated by species from the Calthion alliance and $\mathrm{Mo}$ linietalia order. In contrast, species of fresh meadows have an insignificant share, which is related to abandonment of mowing. The domination of Deschampsia caespitosa, a species that develops intensively on abandoned meadows, is evident. Authors from other Polish regions (Kompata-Bąba \& Bąba 2007, KrYszaK et al. 2007) report that $D$. caespitosa is able to colonise habitats with adverse conditions (flooding, periodic drying, insufficient fertilisation) and cessation of mowing leads to development of phytocoenoses dominated by this species. The phytocoenoses analysed are additionally accompanied by species from the Phragmitetea class. Particularly intensive development of Carex gracilis and, locally, Phalaris arundinacea, which have the role of dominating species, can be noted. Phytocoenoses with a high share of these species are characterised by poor floristic composition. Similarly, as reported by KOTAŃSKA (unpublished data) from the Proszowice Plateau, abandonment of mowing in patches with C. canum promotes overgrowth by Phalaris arundinacea and Phragmites australis.

Unmown phytocoenoses comprise species from the Artemisietea class. KRYsZAK et al. (2007) reported emergence of species from the Artemisietea vulgaris, Agropyretea intermedio-repentis, and Stellarietea media classes on unmown and ungrazed grasslands. In turn, BARABASZ-Krasny $(2002,2011)$ noted that abandonment of management of former meadow and pasture areas in the Przemyśl Foothills led to eutrophication of habitats and formation of phytocoenoses dominated by herb species and herb-shrub communities.

Analysis of the diversity degree based on the Shannon-Wiener index revealed that the mown stands were characterised by a high species diversity $\left(\mathrm{H}^{\prime}=\right.$ 3.003). Despite the higher total number of species in the community, the abandoned phytocoenoses exhibit a lower diversity index $\left(\mathrm{H}^{\prime}=2.717\right)$. This is related to the species coverage and the number of species. In the mown patches, a considerable number of species are characterised by a high abundance value and stability degree IV-V. In turn, the abandoned patches are dominated by sporadically occurring species with an insignificant impact on the structure of the phytocoenoses. Similar results were obtained by authors from other regions of Poland who observed a significant decline 
in species diversity in abandoned communities (BARABASZ-KRASNY 2002, KrYSZAK et al. 2007).

The values of Ellenberg's ecological indicators for temperature and light are similar in both communities. In contrast, the moisture indicator is higher in the abandoned phytocoenoses. This is associated with the increased share of wet habitat species, whose occurrence may be due to the higher moisture of the substrate or subsequent humidification thereof. In turn, the mown patches are characterised by a lower acidity and higher substrate fertility, which may be related to the commonly applied mineral fertilisation of the meadow grasslands.

\section{CONCLUSIONS}

1. Cirsium canum is a rare species in the Wielopolka River valley in the Strzyżów Foothills. It grows in managed and abandoned meadow phytocoenoses.

2. In the study area, C. canum grows on neutral or slightly acidic soils. The species prefers moist habitats and less frequently wetlands or periodically waterlogged habitats. It occurs in insolated, warm, or moderately warm meadow sites.

3. The managed patches exhibit a clear share of species characteristic for wet and fresh meadows, which is related to the regular mowing thereof. In the abandoned patches, the proportion of fresh meadow species (Arrhenatheretalia) is negligible. They are evidently dominated by species from the Calthion alliance and Molinietalia order.

4. Regular mowing, fertilisation, and sowing of arable meadow grasslands inhabited by $C$. canum increase the species richness in these phytocoenoses towards communities from the Arrhenatherion alliance and Arrhenatheretalia order. There is a large share of species from the Molinio-Arrhenatheretea class, which achieve a high abundance value, and therefore they cannot be clearly classified into a specific association.

5. The floristic composition of the meadow communities with C. canum is clearly influenced by the management, which determines the trend of vegetation development of these phytocoenoses.

\section{REFERENCES}

BARABASZ-Krasny B. (2002): Sukcesja roślinności na łąkach, pastwiskach i nieużytkach porolnych Pogórza Przemyskiego. Fragmenta Floristica et Geobotanica Polonica, Supplementum 4: 1-81.

BARABASZ-Krasny B. (2011): Zróżnicowanie roślinności i sukcesja wtórna na odłogach wielkopowierzchniowych Pogórza Przemyskiego. W. Szafer Institute of Botany, Polish Academy of Sciences, Kraków.
Ellenberg H., Weber H.E., Düll R., Writh V., Werner W., Paulissen D. (1992): Zeigerwerte von Pflanzen in Mitteleuropa. Scripta Geobotanica 18: 1-258.

FijąKowski D., CHojnaCKA-FijaŁKowsKa E. (1990): Zbiorowiska z klas Phragmitetea, Molinio-Arrhenatheretea i Scheuchzerio-Caricetea fuscae $\mathrm{w}$ makroregionie lubelskim. Roczniki Nauk Rolniczych, Seria D, z. 217.

Kompata-Bąba A., Bąba W. (2007): Przemiany składu florystycznego zbiorowisk łąkowych Kotliny Dąbrowskiej (Wyżyna Śląska) jako wynik zaprzestania tradycyjnych form użytkowania i degradacji środowiska. Acta Botanica Warmiae et Masuriae 4: 173-186.

KondRaCKI J. (2011): Geografia regionalna Polski. Wyd. Nauk. PWN, Warszawa.

KotaŃSKa M., Towpasz K. (2007): Zbiorowiska łąkowe z Cirsium canum (L.) All. na Płaskowyżu Proszowickim (Wyżyna Małopolska). In: E. Kępczyńska, J. Kępczyński (eds). Botanika w Polsce - sukcesy, problemy, perspektywy. Streszczenia referatów i posterów. 54. Zjazd PTB, Szczecin: 91. Wyd. Oficyna In Plus.

Krebs J.CH. (2011): Ekologia. Wyd. Nauk. PWN, Warszawa.

Kryszak A., KrysZak J., Grynia M. (2007): Zmiany degradacyjne na łąkach i pastwiskach wyłączonych $z$ użytkowania. Acta Botanica Warmiae et Masuriae 4: 205-214.

Matuszkiewicz W. (2005): Przewodnik do oznaczania zbiorowisk roślinnych Polski. Wyd. Nauk. PWN, Warszawa.

Meusel H., JäGer E.J. (1992): Vergleichende Chorologie der Zentraleuropäichen Flora. 3. Fischer, Jena-Stuttgart-New York.

Mirek Z., Pięroś-Mirkowa H., Zając A., Zając M. (2002): Flowering plants and pteridophytes of Poland. A checklist. Vol. 1. Biodiversity of Poland. - Krytyczna lista roślin naczyniowych Polski. T. 1. Różnorodność biologiczna Polski. W. Szafer Institute of Botany, Polish Academy of Sciences, Kraków.

PAwŁowski B. (1977 a): Skład i budowa zbiorowisk roślinnych oraz metody ich badania. In: W. Szafer, K. Zarzycki (eds). Szata roślinna Polski. Vol. 1. PWN, Warszawa: 237-269.

PAwŁowski B. (1977 b): Szata roślinna gór polskich. In: W. Szafer, K. Zarzycki (eds). Szata roślinna Polski. Vol. 2. PWN, Warszawa: 189-252.

RutKowski L. (2004): Klucz do oznaczania roślin naczyniowych Polski niżowej. Wyd. Nauk. PWN, Warszawa.

TowPAsz K. (1987): Rośliny naczyniowe Pogórza Strzyżowskiego. Zeszyty Naukowe Uniwersytetu Jagiellońskiego, Prace Botaniczne 16.

TowPASz K. (1990): Charakterystyka geobotaniczna Pogórza Strzyżowskiego. Zeszyty Naukowe Uniwersytetu Jagiellońskiego, Rozprawy habilitacyjne 178. 
VichereK J. (1962): Typy fytocenóz aluviální nivy dolního Podyjí se zvláštním zaměřením na společenstva luční. Folia Př́rodovědecké fakulty UJEP v Brně, Biologia, sv. 3, spis 5: 1-113.

ZAJĄC A., ZaJĄC M., eds (2001): Distribution atlas of vascular plants in Poland. Laboratory of Computer Chorology, Institute of Botany, Jagiellonian University, Kraków.
ZająC M., ZająC A. (2009): The geographical element of native flora of Poland. Laboratory of Computer Chorology, Institute of Botany, Jagiellonian University, Kraków.

Zarzycki K., Trzcińska-Tacik H., Różański W., SzeLĄG Z., WoŁek J., Korzeniak U. (2002): Ecological indicator values of vascular plants of Poland. W. Szafer Institute of Botany, Polish Academy of Sciences, Kraków. 